\title{
Calculating a Tachometer Signal From Onboard a Smart Vibration Sensor
}

\author{
Eric Bechhoefer ${ }^{1}$, and David $\mathrm{He}^{2}$ \\ ${ }^{1}$ GPMS Inc., Cornwall, VT 05753 \\ eric@gpsm-vt.com \\ ¿University of Illinois at Chicago, Department of Mechanical and Industrial Engineering, Chicago, IL, 69697, USA \\ davidhe@uic.ed
}

\begin{abstract}
Because of the bandwidth limitation of the engine controller, all helicopter gearboxes change speed over time. This change in speed necessitates the resampling of the data, based on a tachometer signal, to facilitate shaft, gear and bearing analysis of the rotating equipment. Without resampling, the quality of vibration analysis is degraded, and many mechanical faults would be missed. Further, interfacing with existing tachometer can be both expensive, and in some cases change certification requirements. The ability for a smart sensor to acquire vibration data, extract the shaft speed from the vibration data, and then process the data allows vibration based fault detection capability at a lower cost, weight and reduced installation complexity than previously possible. Reducing cost, weight and installation complexity will expand the business case for condition monitoring, improving safety and reliability in industrial and transportation systems. This paper demonstrates a twostep process to recover a tachometer signal from vibration data that is of higher quality than raw tachometer data. Statistics are generated from known fault cases to demonstrate the effectiveness.
\end{abstract}

\section{INTRODUCTION}

R.M Stewart (Stewart 1977) introduced the time synchronous average (TSA) into gearbox analysis and revolutionized condition-based maintenance on helicopters. His ideas, such as FM0/1/2/3/4, the "difference signal", the envelope analysis, etc., is used by most, if not all, Health and Usage Monitoring Systems (HUMS) currently available. Stewart's design is heavily dependent on a

\footnotetext{
Eric Bechhoefer et al. This is an open-access article distributed under the terms of the Creative Commons Attribution 3.0 United States License, which permits unrestricted use, distribution, and reproduction in any medium, provided the original author and source are credited.
}

tachometer input to allow resampling of vibration data for the analysis. Further, Stewart recognized the importance of tachometer jitter, noting that there was a limit to the number of revolutions to take on the TSA (e.g., at some point, the TSA was not consistent).

The need to interface with a tachometer will add cost and complexity to every application. Further, there may be cases, such as glandless pumps (Boiler Circulator Pumps), where due to heat and pressure it is impractical or infeasible to install a tachometer sensor. In other applications, such as monitoring gas turbine engine, interfacing with the existing tachometer for the power turbine or compressor turbine, may change certification requirements (adding cost) and increase system cost and weight. These issues may make adding a tachometer impractical. Hence, the ability to derive a tachometer signal from vibration can reduce both cost and weight, and may facilitate new application where it is impractical to integrate a tachometer.

For helicopter applications, weight and cost of a HUMS integration can be reduced through the use of a bused, smart system (Bechhoefer, 2012). The use of a data bus dramatically reduces the interconnect weight, while smart sensor makes for a more scalable system. The smart vibration sensor also allows a path to locally processing vibration data to output condition indicators and a tachometer signal.

\section{TACHOMETER SigNAL FROM VibRATION}

The ability for a smart sensor to acquire vibration data, extract the shaft speed from the vibration data, and then process the data allows vibration based fault detection capability at a lower cost, weight and reduced installation complexity than traditional tachometer based system. Reducing cost, weight and installation complexity will expand the business case for condition monitoring, 
improving safety and reliability in light helicopters. Cost is an essential driver in the decision for operators to implement a HUMS. It is well recognized that HUMS improves operational availability and reduces maintenance cost (through an installed Rotor Track and Balance function, opportunistic maintenance, exceedance monitoring, etc.). However, the decision to install a HUMS for many operators is driven by cost. Removing a tachometer sensor and interface, and adding this function to a smart sensor improves the value of a system to a customer.

The spectral content of vibration is measured using the Fast Fourier Transform (FFT). The FFT is used in vibrationbased diagnostics to determine the magnitude and phase of components vibration (such as shafts, gears or bearings), which can be indicative or wear and failure. Additionally, many common vibration analyses (Residual or Difference Analysis, Narrowband analysis (Lebold, 2000, McInerny, 2004) use the FFT for ideal filtering of the signal, or to perform a Hilbert transform of the signal (Amplitude and Frequency Analysis).

The Fourier transform (and FFT) assumes that the signal under analysis is infinite in time. This assumption fails for real signals but can be mitigated by the use of a window function (such as a Hamming or Hanning window). The other assumption in using the FFT is that the signal is stationary. Stationarity implies that the conditions of the signal do not change, e.g., the shaft rate is constant. The assumption in practice is violated because rotating machines have variation in their shaft rate. This change in rotational rate is due to changing load and the limits of the feedback control bandwidth.

In practice, the failure of stationarity results in "spectral smearing" of energy associated with a shaft. Spectral smearing, in turn, results in not measuring, accurately, the energy associated with a particular fault frequency. To improve the performance of analysis using the FFT, Time Synchronous Averaging (the TSA, for shaft/gear analysis) and Time Synchronous Resampling (TSR) have been developed (figure 1).

\subsection{Resampling Algorithms}

The TSA resamples the vibration associated with a shaft or gear, in the spatial domain; such that vibration associated with each shaft order, in the Fourier domain, represent one frequency bin. For example, consider a system in which the shaft rate is such that for a given vibration sample rate the acquisition system on average collects 800 samples per revolution. The TSA would resample the 800 samples to the 1024 data points. The value 1024 is the next highest radix 2 value. Radix 2 values are used because the most straightforward implementation of the FFT is based on powers or 2, e.g., radix 2 values.
Consider that the load on the shaft reduces, the shaft rate increases and the number of measured vibration samples is 780. It takes less time for the shaft to make one revolution, hence the fewer sample rate. The 780 samples are resampled to 1024 points. At some future time, the load on the shaft may increase, slowing the shaft, resulting in measuring 820 samples, again the data is resampled to 1024 points. For every revolution, the resampled data is summed point by point. After $n$ revolutions, each of the 1024 points of is divided by $n$, essentially time synchronously averaging the vibration data

Assume in this case that there is a gear with 37-tooth gear on the shaft. The gear mesh energy of the gear would be a frequency of $37 \mathrm{x}$ the shaft rate. In the Fourier domain, the energy associated with the shaft rate would be in bin 2, and the gear mesh energy would be bin 38, and the second harmonic of that gear would be in bin $75(37 \times 2+1$, bin 1 is the DC energy). The TSA also reduces non-synchronous vibration by $1 / \operatorname{sqrt}(n)$, where $n$ is the total number of shaft revolutions which were used to construct the TSA. In this way, the TSA corrects for variation in the shaft rate and improves the signal to noise.

The TSR similarly resamples (e.g., upsamples) the vibration to correct variation in shaft speed. The apparent sample rate is the ratio of the total resampled time domain; vibration data set length divided by original data set length, multiplied by the original sample rate.

Both the TSA and TSR use a tachometer signal to calculate the time over which a shaft completes one revolution. The time taken for any shaft to complete a rotation can be calculated even if the tachometer is not associated with a given shaft. This is done by taking into account the shaft ratio from the shaft with the tachometer, to the shaft under analysis, then interpolating based on that tachometer signal.
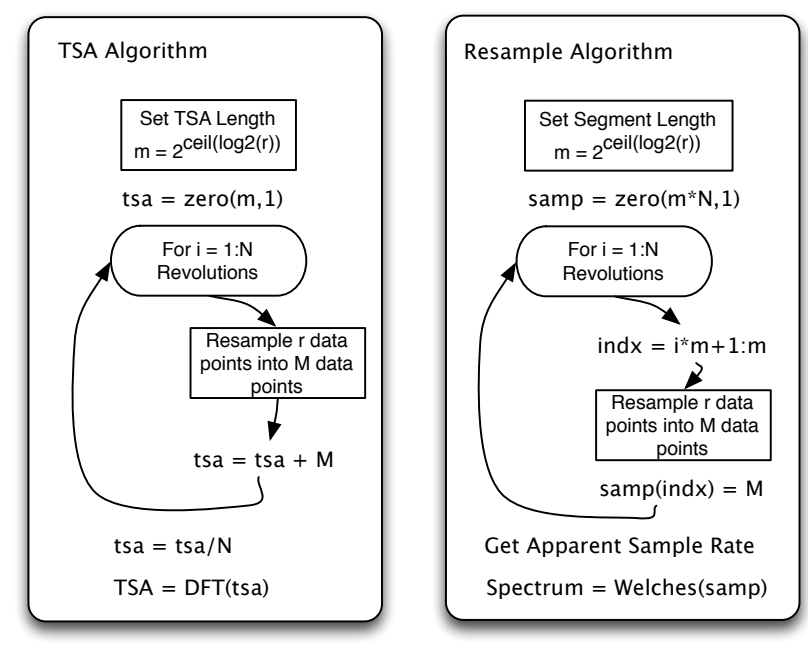

Figure 1 Resampling Algorithms for Vibration-Based Diagnostics 


\subsection{Tachometer Signal Measurement}

In implementation, the tachometer signal is the rising edge of a voltage trigger from the passing of a shaft key phasor (e.g., a stationary point of the shaft). The tachometer signal is then converted to time. This time is accrued for each pass of the key phasor. In an architecture where the tachometer signal is recorded using an analog to digital converter (ADC), the resolution in time of the rising edge is 1 over the sample rate of the ADC. For condition monitoring purposes, the sample rate for a high-speed shaft would be 100,000 samples per second. In another architecture, the tachometer signal inputs into a voltage comparator. When the tachometer signal crosses zero (or some low voltage offset), the comparator goes high. The output of the comparator is monitored by the microcontroller using a general purpose input/output (GPIO) pin. When the microcontroller senses the GPIO pin going high, it records the time. The resolution of time on the microcontroller is typically much higher than an ADC. For example, in a system using a $12 \mathrm{MHz}$ clock, the microcontroller might run at $96 \mathrm{MHz}$, but the counter for time in the microcontroller would run at $48 \mathrm{MHz}$. The tachometer resolution in time would then be $2.0822 \mathrm{e}-8$ seconds.

Vibration signals from rotating equipment are sinusoidal and, by definition, synchronous with the shaft. However, the nature of vibration makes it impossible to use vibration signal without significant signal process. Measured vibration is the superposition (e.g., addition) of many signals in time domain. For example, consider a simple gearbox with an input shaft, an output shaft and a gear pair. The input shaft turns at $30 \mathrm{~Hz}$, and has a 32 tooth gear, the output shaft has a 82 tooth gear with a speed of $11.707 \mathrm{~Hz}$. The gear mesh frequency is $30 * 32=960 \mathrm{~Hz}$. It is likely that the gear mesh frequency will have sidebands as a result of any shaft imbalance being modulated onto the gear mesh. This can be proved using the trigonometric identity:

$$
\cos (a) * \cos (b)=1 / 2[\cos (a+b)+\cos (a-b)]
$$

Where in this example, $\cos (a)$ is $960 \mathrm{~Hz}$, and $\cos (b)$ is 30 and/or $11.707 \mathrm{~Hz}$ shaft. Additionally, if the shaft is bent or bowed, there will be a $2 \mathrm{x}$ shaft vibration component. Other manufacturing defects such as the gear not being mounted perpendicular to the shaft, or not centering the shaft on the gear (e.g. eccentricity) adds additional tones.

\section{TACHOMETER SIGNAL FROM VIBRATION}

Bonnardot in 2005 describes a method of using an acceleration signal of a gearbox to perform the TSA. Tachless TSA was further developed by Combet in 2007. These methods call for bandpass filtering of the signal around a gear mesh, then in Bonnardot, taking the Hilbert transform to estimate phase, and using phase to estimate zero crossing time. In Combet, the signal was bandpass filtered then demodulated, with the time resulting time domain signal used for the zero crossing.

Both solutions are suboptimal, due to the nature of using the FIR (Finite Impulse Response) bandpass filter. Additionally, because modeling errors are present even after bandpass filtering, the tachometer signal is corrupted by jitter. In this paper, we present a two-step method. Step one is to ideally bandpass and creates an analytic signal in one functional procedure. Step two is to use a jitter reduction model to remove noise (jitter) from the reconstructed tachometer signal not associated with changes in machine rate. While this system could be implemented on any computer, it is ideally suited for implementation in a smart sensor, where cost and weight or other issues can be better addressed by a lower cost condition monitoring system.

The pseudo code to recover a tachometer signal from vibration is:

- $\quad$ Define the Sample rate $=s r$. The number of data points of vibration data, $n=s r \mathrm{x}$ acquisition length in seconds, then:

- Calculate the next larger radix-2 length for the FFT. nRadix $=2^{\wedge} \operatorname{ceil}\left(\log _{2}(n)\right)$

- Calculate the low and high bandwidth index (bwlow, bwhigh), which are centered are a known gear mesh

- $\quad$ Take the zero padded FFT of the vibration data

- Zero the FFT from zero to bwlow, and from bwhigh to nRadix

- Take the inverse FFT, generating the analytic signal

- Calculate the unwrapped argument of the signal from 1 to $n$ time series

- Normalize the time series of radians by the number of teeth of the gear (assuming $1^{\text {st }}$ harmonics)

- Interpolate the number of indexes for every $2 \pi$ radians

- Normalized to tachometer zero crossing times by $s r$.

A bandpass filter, which is well known to those skilled in the arts, is the convolution of a low pass filter with a high pass filter. These filters are implemented as Finite Impulse Response (FIR) filters to improve their stability. Unfortunately, even rather large filters have a poor response. Consider a case of a wind turbine gearbox, with those as mentioned earlier approximately $29 \mathrm{~Hz}$ shaft and a 32 tooth gear, or $928 \mathrm{~Hz}$. The bandwidth of the filter is set from $910 \mathrm{~Hz}$ to $950 \mathrm{~Hz}$, to exclude the $30 \mathrm{~Hz}$ of the high speed shaft (e.g. $29 * 32-29=899 \mathrm{~Hz}$ and e.g. $29 * 32+29$ $=957 \mathrm{~Hz}$ ). The filter response for this case, using a 120-tap FIR filter is seen in Figure 2. 


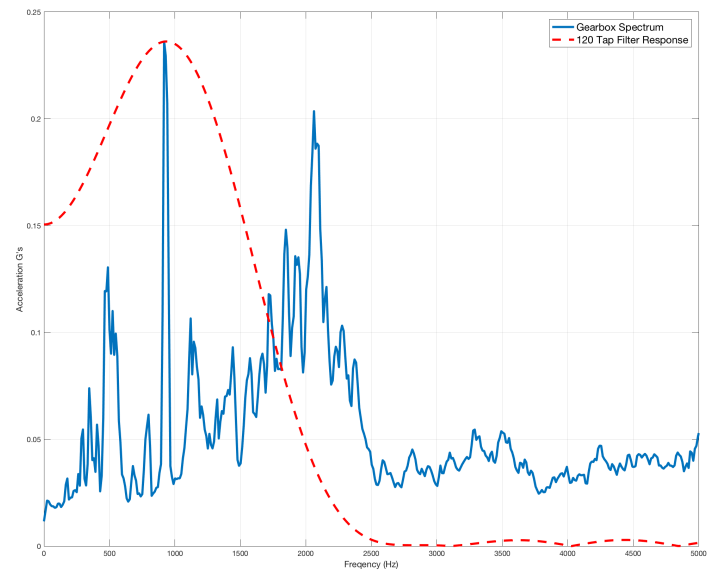

Figure 2 Spectrum from a wind turbine high-speed shaft, and a 120 tap FIR bandpass filter

Note that the bandwidth $(50 \%$ power, $3 \mathrm{~dB})$ of this filter is $1670 \mathrm{~Hz}$. The filter does not reject the spectral content at $500 \mathrm{~Hz}$, or at $1121 \mathrm{~Hz}$. These additional frequencies will be superimposed on the $928 \mathrm{~Hz}$ signal will distort the desired analytic signal, reducing the quality of the resulting tachometer signal.

Consider a process in which developing the analytic signal using an ideal filter is completed in a single functional process. The analytic signal is defined for real the realvalued signal $s(t)$ as $\mathrm{Eq} 2$. :

$$
S(f)=F\{s(t)\}
$$

Where $F$ is the Fast Fourier Transform, where:

$$
\begin{gathered}
S_{a}(f)=S(f), \quad f=0 \\
S_{a}(f)=2 S(f), f>0 \\
S_{a}(f)=0, f<0 \\
S_{a}(t)=F^{-1}\left(S_{a}(f)\right)
\end{gathered}
$$

$S(f)$ is as noted, the Fourier transform of $s(t)$.

For a signal which is sampled at 97656 samples per second, for six seconds, the total length of $s(t)$ is $n, 585936$ data points. As noted, there are advantages to using radix 2 lengths for the Fast Fourier Transform (FFT). By zero padding the FFT to next larger radix two value, $2^{\wedge} 20$ or 1048576, the index representing the cutoff frequency for the bandpass frequencies are: bwlow $=910 \mathrm{~Hz} / 97656 \mathrm{x}$ $1048576=9771$, and $b w h i g h=960 / 97656 * 1048576=$ 10308. Then one can define the bandpass analytic signal in Eq 4:

$$
\begin{gathered}
S_{a}(f)=2 S(f), \text { bwlow } \leq f \leq \text { bwhigh } \\
S_{a}(f)=0, f<\text { bwlow }, f>\text { bwhigh } \\
s_{a}(t)=F^{-1}\left(S_{a}(f)\right)
\end{gathered}
$$

There is no need to multiply by 2 , as the argument (e.g., angle) which is of interest, is the arctangent ratio of the imaginary parts of $s_{a}(t)$ and the real parts of $s_{a}(t)$. The idealized band pass function rejects all signals not associated with the desired passband. Figure 3 is a zoomed view of the spectrum in Figure 2. comparing the passband of the idealized filtered realized using Eq 3.

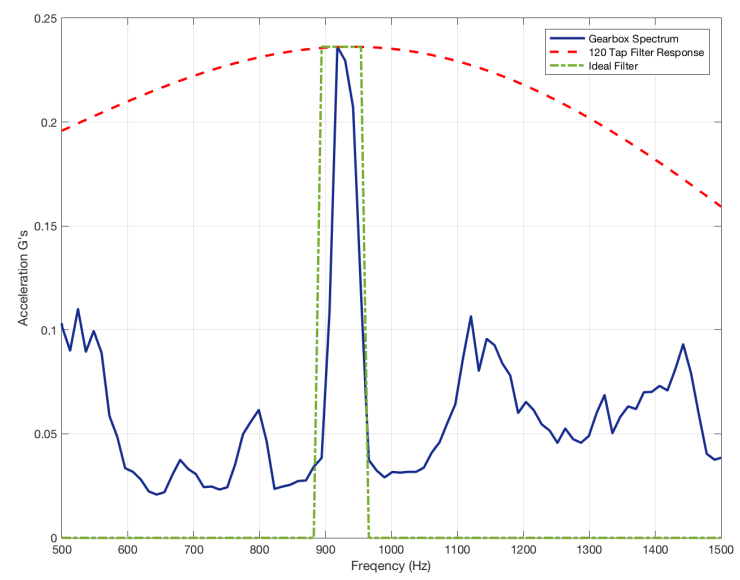

Figure 3 FIR bandpass filter vs. the idealized filtered realized using Eq 4.

The FIR filter does not reject the $500 \mathrm{~Hz}$ and $1150 \mathrm{~Hz}$ tone. The idealized filter captures only the signal associated with the desired gear mesh tone. This idealized filter allows for a higher signal to noise ratio and improved reconstruction of the tachometer signal from vibration. This tachometer signal is recovered from the arctangent of the analytic signal.

Further, the arctangent function returns radians between 0 to $\pi$ and $-\pi$ to 0 . However, the evolution of the angle represents the incremental increase in phase for each sample in time. For example, the phase of the analytic signal for three cycles is $2 \pi \times 3$ or $6 \pi$. The arctangent of that signal will be $-\pi$ to $\pi$ for 3 cycles. The result of the arctangent must be unwrapped to capture in increases phase vs. time. Unwrapping of the phase angle requires keeping track of the previous angle and current angle. The current angle is added to the previous angle, except when the returned arctangent goes from $\pi$ to $-\pi$. In this case, $\pi$ is added to the returned value to correct for the case when the returned value is between $-\pi$ to 0 .

After unwrapping the phase angle, the units are in radians per sample. While the FFT and inverse FFT operated on the radix 2 length (in this case 1048576), the argument and phase angle computation are performed only on the original sample length, $n$. Note that this time series of radians is for the gear mesh. To converts to radians per revolution of the shaft, the time series is divided by the number of teeth in the gear. In this example, 32. 
However, it may be that the most reliable gear mesh tone is the $2^{\text {nd }}$ or $3^{\text {rd }}$ harmonic, in which case the passband is adjusted accordingly, and the time series of radian angle is divided by 2 x number of teeth for the $2^{\text {nd }}$ harmonic.

The resulting time series represents the radian angle of the shaft, where each index advances the angle in time by $d t$, or $1 /$ sample rate. Every $2 \pi$ radians represents one shaft revolution. Because one is interested in the time, precisely every $2 \pi$, a form of interpolation is needed. For example, consider that the index just prior to $2 \pi$ is 6.282780795474 (or 0.0004 less than $2 \pi$ ) at array index 3395, while at index 3396 , the radian value is 6.284629142378 , or 0.0014 greater than $2 \pi$. It is then a simple matter to interpolate between the index 3395 and 3396 for a radian value of $2 \pi$. In this case, the interpolated value is 3395.21885053316 .

This interpolation gives the number of indexes for each revolution. Note that this estimate of the tachometer zero cross signal is corrupted by noise. Combert (2007) reports that the phase error standard deviation is related to the local signal to noise at the mesh harmonic $k$ as:

$$
\sigma[\delta]=1 / \sqrt{2} 10^{-S N R / 20}
$$

Typical measured SNR is 6 to $8 \mathrm{~dB}$. This suggests that the standard deviation of the phase error would be 6 to 10 degrees. This phase is zero mean, but as it is non zero, it will add jitter to the reconstructed tachometer signal.

\subsection{Controlling Tachometer Jitter}

In "Improving Gear Fault Detection by Reducing Tachometer Jitter" (Bechhoefer, 2014), it was shown that tachometer jitter contained by a low a low-frequency component associated with the engine control unit, and random, higher frequency components. Bechhoefer et al. improved gear fault detection by using a zero phase, low order IIR, backward/forward filtering. As noted previously, both FIR and IIR filters bandwidth is defined by the $3 \mathrm{~dB}$ reduction in signal energy. The filter does not remove all signals above the bandwidth, and in fact, reduces some signal energy below the cutoff (up to $50 \%$ ).

The idealized filter using the FFT processing is zero phase as well. The improve analysis proceed similarly to the forward/backward process:

- Take the pseudo derivative of the tachometer signal

- Calculate the radix-2 length of the pseudo derivative signal of length $n$

- Zero pad the array from $n$ to the radix-2 length

- Calculate the bandwidth index of the FFT

- $\quad \mathrm{Idx}=$ floor(bandwidth * radix-2 length / 2);

- Bandwidth is a normalized value, typically 0.12

- Take the real FFT of the zero padded derivative signal
- Set the real and imaginary parts of the FFT from Idx to the radix-2 length

- Take the inverse real FFT.

- Reconstruct the tachometer signal by taking the pseudo integral of the signal

Figure 4 demonstrates the effectiveness of the ideal low pass filter in removing jitter from the tachometer signal. The tachometer was from a wind turbine with 8 targets per revolution.

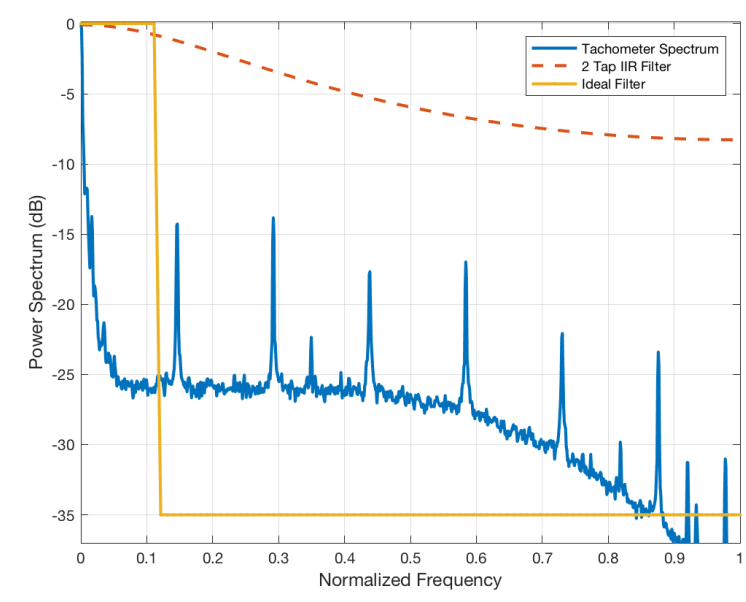

Figure 4 Tachometer pseudo derivative spectrum compared to the IIR and the ideal low pass filter.

Note that the idealized filter retains more low-frequency response than the IIR filter, and effectively removes all spectral power (jitter) above the cutoff frequency.

\section{Numerical CONSIDERATIONS}

For an embedded system, the decision to use float vs. double precision has significant repercussions. The smart vibration sensor developed for this project uses a Renesas microcontroller with a hardware floating point unit and 32 $\mathrm{MB}$ of SDRAM. This gives $4 \mathrm{MB}$ of floating point data. The system was designed around the use of the Real FFT FFT. Hence the scratchpad RAM for analysis is the same size as the input data array. This means the maximum data array is 2,097,152 measurements of vibration data if using float, or 1,048,576 for double precision.

Additionally, because this storage is not on the microcontroller itself, use of double precision numbers take $4 \mathrm{x}$ the clock cycles to move the data from RAM into the register, operate on it, then move the new value back into RAM. It should seem that floating-point numbers would be the default cast.

Consider a real world application where the maximum sample rate $93750 \mathrm{sps}$, for analysis on the turbine shaft of a Rolls Royce m250 engine (power turbine speed of 32,187 RPM and the compressor turbine RPM of 51,000). For a 
two second acquisition, this is 187500 samples or a radix 2 length of 262144 . In the FFT, the radian update angle is then $2 \pi / 262144$ radians, or $2.4 \mathrm{e}-5$, which is the generally seen as being less than the precision of the float. This is why most embedded systems do not perform FFTs greater than length 16384. Numerically, the use of float for the tachometer from vibration algorithm produces unacceptable results (Figure 5).

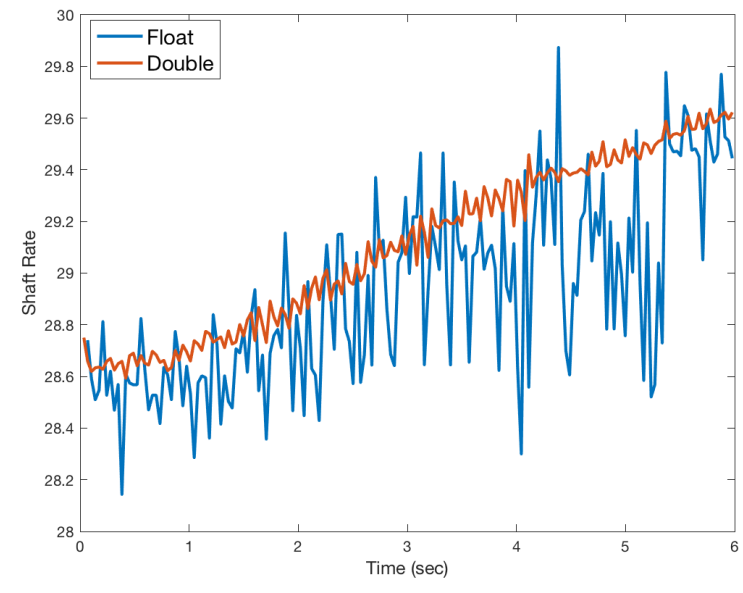

Figure 5 Float vs. Double precision calculation of tachometer from vibration

As noted, using double for the measurement does "fix" the problem, at the expense of very long processing time (going from approximately 3 minutes to 12 minutes on the microcontroller) and cuts in half the maximum amount of data that can be measured. The key then is to measure them and store the data as float, but internal to the FFT calculation, use a double representation of the radian angles. It was found that the compilers in the FPU, promoted floats to double for multiplication and addition. By defining those operands as double, the speed of the calculations was not reduced: the processing speed was almost entirely a function of moving the data from RAM to the register and back to RAM.

\section{High-Speed Pinion on A Wind turbine, CASE 1}

This machine was found, through vibration based diagnostics, to have a cracked tooth on the high-speed pinion. The acquisition length was six seconds, sampled at 97656 samples per second. The high-speed shaft is approximately $30 \mathrm{~Hz}$.

This system was equipped with a tachometer, which is used as a reference to compare against the vibration based tachometer signal. The installed tachometer was a Hall sensor, using the GPIO architecture to capture zero crossing time. The tachometer target had eight pulses per revolution, but time was calculated using each $8^{\text {th }}$ timing mark (e.g., one revolution). Figure 6 compares shaft speed derived from the Hall sensor base tachometer signal, vs. the vibration based tachometer signal, vs. the final processing use the new jitter reduction technique. Wind turbine analysis is particularly tricky in that there is a high degree of variability in the shaft speed. In this example, there is a ramp of approximately $1 \mathrm{~Hz}$. The SNR is $6.7 \mathrm{~dB}$.

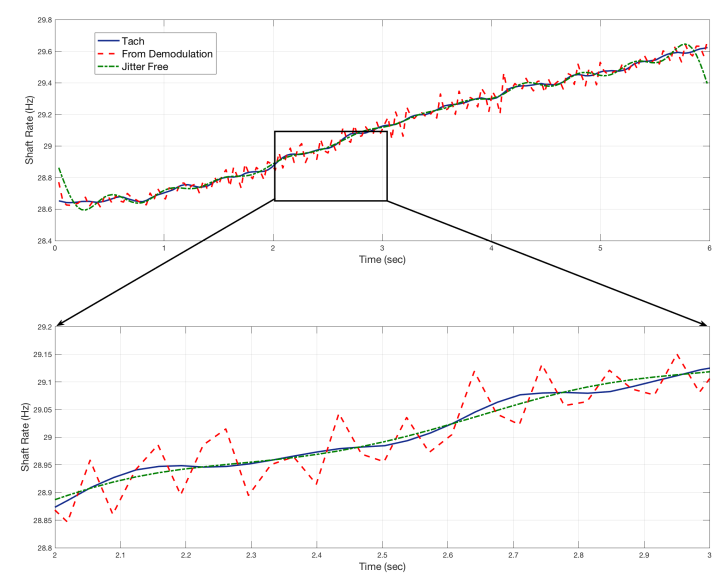

Figure 6 Shaft Rate from Tachometer signal in comparison with jitter-free tachometer from vibration

The bottom graph is a zoomed from the $2^{\text {nd }}$ to $3^{\text {rd }}$ second on the acquisition. In general, after removing jitter using the new process, the vibration based tachometer signal is no significantly difference from the tachometer derived from the Hall sensor. Figure 7 depicts the TSA and the spectrum of the analysis.
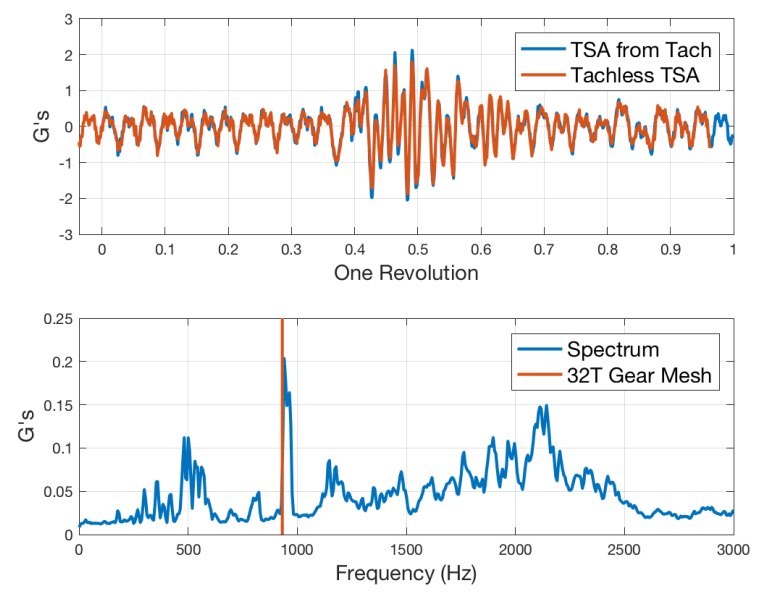

Figure 7 Comparison of the TSA derived from a tachometer and vibration sensor.

The difference between the TSA is effectively only phase angle. Statistics derived from the TSA are given in table 1. 
Table 1 Tach vs. Tach for Standard Vibe Statistics

\begin{tabular}{lll}
\hline Analysis & Tach & Tach from Vibe \\
\hline SO1 & $0.0100 \mathrm{~g}$ & $0.0104 \mathrm{~g}$ \\
\hline SO2 & $0.0013 \mathrm{~g}$ & $0.0016 \mathrm{~g}$ \\
\hline SO3 & $0.0019 \mathrm{~g}$ & $0.0018 \mathrm{~g}$ \\
\hline TSA RMS & $0.5091 \mathrm{~g}$ & $0.4828 \mathrm{~g}$ \\
\hline TSA P2P & $2.0887 \mathrm{~g}$ & $1.8430 \mathrm{~g}$ \\
\hline FM0 & 4.278 & 4.00 \\
\hline AM RMS & $0.100 \mathrm{~g}$ & $0.099 \mathrm{~g}$ \\
\hline AM Kurtosis & 4.242 & 4.217 \\
\hline FM RMS & 0.428 radians & 0.426 radians \\
\hline FM Kurtosis & 4.995 & 4.844 \\
\hline
\end{tabular}

The gear fault was detectable with either a tachometer or via vibration method.

\section{TAil Rotor InTERmediate Gearbox Pinion Fault, CASE 2}

This was taken from public domain data set, from a test stand. The sample rate was 100000 samples per second, using the ADC architecture for recording zero crossing data. The tachometer sensor was a variable reluctance speed sensor, using a 22 tooth gear as a target. The shaft rate for the target gear was approximately $3000 \mathrm{rpm}$ (e.g., gear mesh frequency of $500 \mathrm{~Hz}$ ), with a ratio from this shaft to the tail rotor drive shaft of 7.3:1. As seen in Figure 8, the tachometer signal is noisy (high jitter), with a tail rotor shaft rate of $68.5 \mathrm{~Hz}$. The zoomed view shows the tachometer from vibration and with less jitter than the VR sensor tachometer signal, while the jitter-free tachometer signal is smooth.

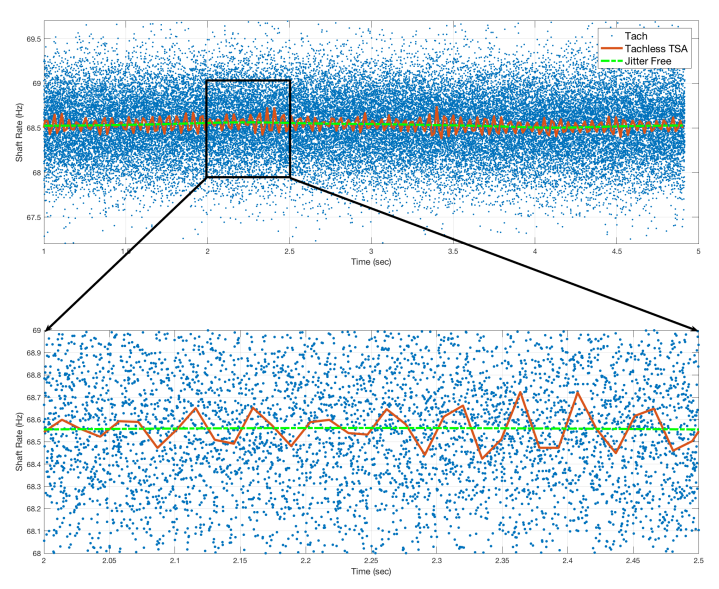

Figure 8 VR Tachometer vs. tach and jitter-free tach from vibration

The SNR for this example is approximately 12 ; due to the $65 \mathrm{~g}$ mesh frequency (Figure 9). The resulting TSA, aside from a difference in phase, is indistinguishable from the TSA using a tachometer from the VR sensor.
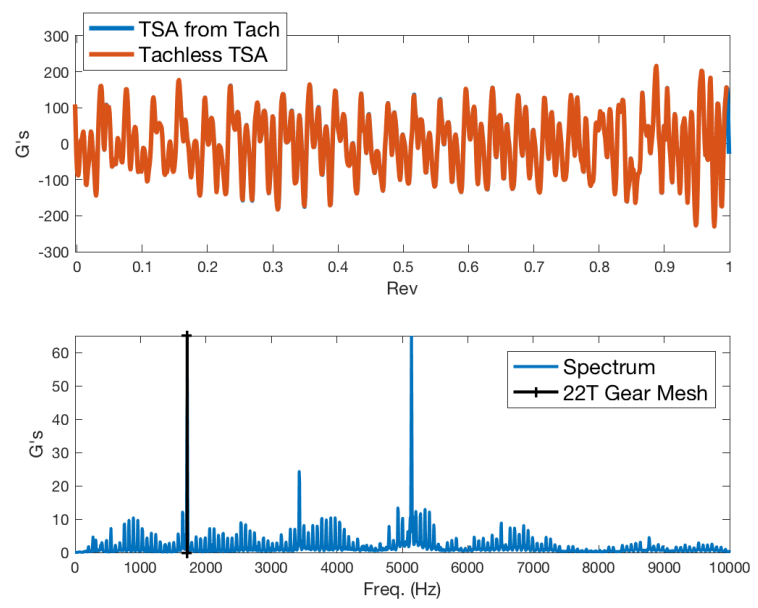

Figure 9 Comparison of the TSA derived from a tachometer vs. vibration sensor

Statistics derived from the TSA for the tail rotor input gear are given in table 2:

Table 2 Tach vs. Tach for Standard Vibe Statistics

\begin{tabular}{lll}
\hline Analysis & Tach & Tach from Vibe \\
\hline $\mathrm{SO} 1$ & $0.043 \mathrm{~g}$ & $0.043 \mathrm{~g}$ \\
\hline $\mathrm{SO} 2$ & $0.283 \mathrm{~g}$ & $0.282 \mathrm{~g}$ \\
\hline $\mathrm{SO} 3$ & $1.855 \mathrm{~g}$ & $1.85 \mathrm{~g}$ \\
\hline TSA RMS & $81.646 \mathrm{~g}$ & $81.591 \mathrm{~g}$ \\
\hline TSA P2P & $222.77 \mathrm{~g}$ & $223.02 \mathrm{~g}$ \\
\hline FM0 & 16.94 & 15.38 \\
\hline AM RMS & $17.06 \mathrm{~g}$ & $17.06 \mathrm{~g}$ \\
\hline AM Kurtosis & 4.126 & 4.126 \\
\hline FM RMS & 3.037 radians & 3.048 radians \\
\hline FM Kurtosis & 2.46 & 2.45 \\
\hline
\end{tabular}

\section{CONCLUSION}

A method to reconstruct the tachometer signal from vibration was demonstrated using a two-step process. The first step uses an idealized bandpass filter implemented with a complex FFT to create the necessary and sufficient analytic signal to need to derive the tachometer signal for the shaft under analysis. The second step uses another idealized low pass filter on the pseudo derivative of the reconstructed tachometer signal to remove jitter. This processing is run on a smart vibration sensor, which facilitates improved vibration analysis on rotating equipment where in the past the addition of a tachometer would be prohibitive due to cost, weight, certification requirements or physically impractical.

The quality of the resulting analysis compares favorably with traditional tachometers on real-world fault data. In fact, 
the tachometer from vibration signal may have less noise, resulting in improved fault detection, than traditional tachometer sensor-based system.

\section{REFERENCES}

Bechhoefer, E., Augustin, M, Kingsley, M., "Architecture for a Light Helicopter HUMS," AHS Forum 68, May 1, 2012.

Bechhoefer, E., He, D., "Improving Gear Fault Detection by Reducing Tachometer Jitter," AHS Forum 72, May 1, 2014.

Bechhoefer, E., Van Hecke, B., He, D., Processing for Improved Spectral Analysis, PHM Society Annual Forum, 2013.

Bonnardot, F., El Badaoui, M., Randal, R. B., Daniere, J., Guillet, J., "Use of the acceleration signal of a gearbox in order to perform angular resampling (with limited speed fluctuations)," Mechanical Systems and Signal Processing 19 (2005) 766-785.

Combet, F., Gelman, L., "An automated methodology for performing time synchronous averaging of a gearbox signal without speed sensor," Mechanical Systems and Signal Processing 21 (2007) 2590-2606.

Lebold, M., McClintic, K., Campbell, R., Byington, C., Maynard, K., "Review of Vibration Analysis Methods for Gearbox Diagnostics and Prognostics," Proceedings of the 54th Meeting of the Society for Machinery Failure Prevention Technology, 2000.

McInerny, S.A., Sun, Q., Hardman, B., "Public Domain Algorithms for Bearing and Gear Fault Detection,"
Proceedings of the 11th International Conference on Structure Borne Sound and Vibration, 2004.

Stewart, R.M., "Some Useful Data Analysis Techniques for Gearbox Diagnostics," Institute of Sound and Vibration Research, Proceeding of Meeting on Application of Time Series Analysis, Sept. 1977

\section{BIOGRAPHIES}

Eric Bechhoefer received his B.S. in Biology from the University of Michigan, his M.S. in Operations Research from the Naval Postgraduate School, and a Ph.D. in General Engineering from Kennedy Western University. He is a former Naval Aviator who has worked extensively on condition-based maintenance, rotor track and balance, vibration analysis of rotating machinery and fault detection in electronic systems.

David He received his B.S. degree in metallurgical engineering from Shanghai University of Technology, China, MBA degree from The University of Northern Iowa, and a Ph.D. degree in industrial engineering from The University of Iowa in 1994. Dr. He is a Professor and Director of the Intelligent Systems Modeling \& Development Laboratory in the Department of Mechanical and Industrial Engineering at The University of IllinoisChicago. Dr. He's research areas include machinery health monitoring, diagnosis and prognosis, complex systems failure analysis, quality and reliability engineering, and manufacturing systems design, modeling, scheduling, and planning. 